\title{
Modeling of shock absorption in athletics track surfaces
}

Luca Andena ${ }^{1}$, Francesco Briatico-Vangosa ${ }^{1}$, Emanuele Cazzoni ${ }^{1}$, Antonio Ciancio ${ }^{1}$, Stefano Mariani ${ }^{2}$, Andrea Pavan ${ }^{1}$.

${ }^{1}$ Politecnico di Milano, Dipartimento di Chimica, Materiali e Ingegneria Chimica “Giulio Natta”, Piazza L. Da Vinci, 32, Milano (Italy)

${ }^{2}$ Politecnico di Milano, Dipartimento di Ingegneria Civile e Ambientale, Piazza L. Da Vinci, 32, Milano (Italy) 


\begin{abstract}
In this work the possibility of predicting the Force Reduction $(F R)$ characterizing the shock absorption capability of track surfaces by finite element modeling was investigated. The mechanical responses of a typical sport surface and of a reference material were characterized by quasi-static uniaxial compression experiments and fitted by Neo-Hookean and Mooney-Rivlin's hyperelastic models to select the more appropriate one. Furthermore, in order to examine the materials behavior at strain rates typical of athletics applications, the rate dependence of the constitutive parameters was investigated. A finite element model, taking into consideration the post-impact nonlinear dynamics of the track surface and of the system (track surface + artificial athlete), was developed and validated through comparison with the results of FR tests. The simulations showed a very good agreement with the experiments and allowed to interpret the experimentally observed combined effect of track thickness and material intrinsic properties on the overall surface behavior.
\end{abstract}




\section{Introduction and aim of the work}

The widely acknowledged ability [1-2] of polymeric materials to absorb the shocks by reducing the amplitude of the shock waves travelling through the human locomotion system makes them especially suitable as shock absorbers in sports applications. Considering athletics, a significant body of literature debates about the effects of shoe and track surface materials on running or jumping impacts, focusing primarily on the athletes' physiology and the prevention of sports injuries [3-7]. The contribution of materials engineering to the design of products that can reduce the risk of such injuries while securing high performance, has been relatively minor; only a few studies on system dynamics and energy aspects have been performed with computer aided modeling [8-11]. Obviously, such studies cannot neglect the properties of the constituent polymeric materials and the structure of the tracks, as different stress levels can be reached during impact on the surface on varying the characteristics of the shoe [11] and of the surface itself [6].

Focusing on running track surfaces, nowadays they are paved in-situ, by laying the admixture of raw materials directly on the substrate, or prefabricated and subsequently bounded to the substrate with appropriate adhesives [12-13]. The materials used span from cast polyurethane elastomers to resin-bound rubber crumbs and calender filled synthetic rubbers.

The tests used to characterize the tracks behavior can be categorized into two groups: those gathering the intrinsic mechanical properties of the constituent materials and those collecting quantities measured directly on the manufactured track by a drop test mimicking the running action. Of the two categories, the former is surely more appealing for designing and developing new surfaces, while the latter is more directly related to the track performance and safety. The International Association of Athletics Federation (IAAF) has promoted the latter, and adopted two standardized tests for the approval of track surfaces [12-15]. One test is referred to as the Force Reduction $(F R)$ test [15]: it is performed with the so-called artificial athlete, an instrument, sketched in Figure 1, that attempts to reproduce the impact of the athlete's heel on the surface. The Force Reduction $(F R)$ output is conventionally defined as 
$F R=\left(1-\frac{F_{\text {max }}}{F_{\text {max }, \text { ref }}}\right) \times 100$

where $F_{\max }$ and $F_{\max , \text { ref }}$ are the peaks in the force-time records measured in two identical impact tests on the track surface $\left(F_{\max }\right)$ and on a conventionally hard surface $\left(F_{\text {max,ref }}\right)$.

For on-field tests, the substrate is the actual foundation laid to accommodate the track surface and may vary from installation to installation. The characteristics of the substrate are not specified by the standard, which nevertheless mentions that they may affect the $F R$ measurements. For laboratory testing, the substrate is a concrete floor whose force response is to be in accordance with specifications set by the standard.

In the technical and scientific literature there is little agreement, not to say awareness, as to how the $F R$ depends on material properties, structure and thickness of the surface, and nature of the substrate $[9,16]$. Focusing on the surface constituent materials, it is interesting to seek a correlation between $F R$ and their intrinsic mechanical properties, as this would help selecting or developing optimal surfaces for sport applications. Moreover, a better knowledge of that correlation would allow predicting how the material behavior may vary with weather conditions, specifically with temperature and humidity, two parameters that may change considerably during use.

On this topic, Durà et al. [16] investigated the correlation between the intrinsic viscoelastic behavior of three materials used for sport surfaces and their $F R$. Their results show how, following IAAF's and European standards [12-15], it is possible to obtain the same $F R$ with materials having different values of loss factor and dynamic rigidity, which were taken as a measure of the materials' intrinsic damping ability and stiffness, respectively. In particular, an almost identical value of $F R$ could be achieved by combining a high rigidity value with a high loss factor value or, conversely, low values of both. The authors concluded that the two properties, rigidity and loss factor, have opposite effects on $F R$.

In a recent work, Benanti et al. [17] revised that of Durà and co-workers, emphasizing the prominent influence on $F R$ of surface thickness, which dominates over that of the constituent 
materials' properties. This is particularly true in the typical range of surface thickness values - i.e. between $10 \mathrm{~mm}$ and $20 \mathrm{~mm}$ - while for higher thicknesses $F R$ tends to an asymptotic value, which inversely correlates with dynamic rigidity. Instead, no clear correlation was found between the limiting $F R$ value and the dissipative properties of the materials, as characterized by the loss factor measured in Dynamic Mechanical Analysis (DMA). This observation led the authors to conclude that the elastic behavior of the constituent material suffices to determine the athletics track cushioning ability - as characterized by $F R$.

Despite highlighting the combined effects of track surface thickness and constituent material inherent rigidity on $F R$, no simple predictive model was proposed in [17]; yet, the need of an adequate model was pointed out. Besides the patent advantages that such a model would offer in the design and development of optimized track surfaces, it could also help to get a better insight in the dynamics of the impacts occurring during athletics activity, thus supplying important information that goes beyond $F R$ alone to biomechanical studies. This would allow, inter alia, to interpret occasional lack of correlation between track qualification tests and subject tests remarked by Nigg and Yeadon [18] in their detailed review. Moreover, once validated the model could be further improved by including the interaction between track surface and running shoes.

Moving from the above considerations this work aims at checking the possibility of predicting $F R$ of track surfaces by finite element modeling using non-linear elastic (hyperelastic) Neo-Hookean or Mooney-Rivlin's constitutive models [19] to describe the mechanical behavior of the constituent materials.

\section{Materials and experimental methods}

Two of the materials already investigated in [17] were chosen for the present study. They are a running track based on Ethylene-Propylene terpolymer rubber (EPDM) (referred to as material A here and in [17]), whose structure is shown in Figure 2, and a $75 \mathrm{phr}$ (equivalent to a filler volume fraction of 0.29) carbon black filled natural rubber (NR) having a SHORE A hardness of 75. While 
the sample of the former was extracted from an unstructured paved-in running track, the latter is not

normally used for sports surfaces: it was selected here as a useful reference homogeneous material with an overall mechanical behavior similar to material A. The apparent densities of the two materials were determined by weighing cubic samples of known size: they were $640 \mathrm{~kg} / \mathrm{m}^{3}$ and $1400 \mathrm{~kg} / \mathrm{m}^{3}$ for material A and NR, respectively.

The uniaxial compression behavior of the materials was characterized in quasi-static tests, carried out with an 1185-R5800 Instron electromechanical dynamometer. Cubic samples of initial height $L_{0}$ (with $L_{0}=16 \mathrm{~mm}$, the thickness of the track surface in the case of material A, $L_{0}=8 \mathrm{~mm}$ for NR), were compressed up to stretches $\lambda=L / L_{0}$ ( $L$ being the current height of each sample) as high as $\cong 0.1$ for material $\mathrm{A}$ and $\cong 0.5$ for $\mathrm{NR}$ (see also Section 5.1 ). Crosshead speeds corresponding to nominal stretch rates ranging between $0.005 \mathrm{~s}^{-1}$ and $0.6 \mathrm{~s}^{-1}$ were used in order to assess the rate dependence of the nonlinear mechanical responses.

A CEAST Fractovis drop weight impact tester fitted with an instrumented hemispherical punch was used to test the impact response of material A under several impact energies (1.7-13.2 J) and speeds (1.04-1.80 m/s). To avoid specimen perforation or even excessive deformation of the material (with respect to values occurring in the impacted region under the artificial athlete), for these tests the samples were backed by a $5 \mathrm{~mm}$ thick High Density Polyethylene (HDPE) plate. Relative motion between the specimen and the backing plate was suppressed by sandwiching the sample between the HDPE substrate and a steel plate and applying a clamping force of $F_{\mathrm{c}}=984 \mathrm{~N}$.

FR measurements were performed on both materials according to EN 14808 standard [14], using 400x400 $\mathrm{mm}^{2}$ samples laid on a concrete substrate and using an artificial athlete Berlin manufactured by IST Switzerland equipped with a Keithley analyzer. To study the effect of sample thickness, samples of varying thickness were obtained by stacking several layers of the same thickness on top of each other, up to a total thickness larger than $120 \mathrm{~mm}$. No adhesive was used 
between the layers, since measurements performed on samples made of either bound or unbound

\section{Constitutive equations}

Keeping in mind the results obtained in [17] and the expected finite strains rubbery behavior of the materials investigated in this work, the simple yet accurate Neo-Hookean and Mooney-Rivlin's hyperelastic models [19] were considered.

The two models can be formulated in terms of strain energy dependence on Cauchy-Green deformation tensor [20]; however, in order to fit the experimental data of the uniaxial compression tests, the two constitutive models are more conveniently expressed here in terms of the nominal or first Piola-Kirchhoff stress, $\sigma$, as a function of stretch, $\lambda$, as follows:

$$
\sigma^{N H}=2 C_{10}\left(\lambda-\frac{1}{\lambda^{2}}\right)
$$

and

$$
\sigma^{M R}=2\left(C_{10}+\frac{C_{01}}{\lambda}\right)\left(\lambda-\frac{1}{\lambda^{2}}\right)
$$

where the NH and MR stand for Neo-Hookean and Mooney-Rivlin, respectively, and $C_{10}$ and $C_{01}$ are constants. Equation 2 and 3 hold under the hypothesis of material incompressibility. It is worth noting that, in the case of small strains, the linear elastic solution is recovered with $2 C_{10}=G=E / 3$ for the Neo-Hookean model and $2 C_{10}+2 C_{01}=G=E / 3$ for the Mooney-Rivlin's one, where $G$ and $E$ are, respectively, the material shear and Young's moduli.

\section{Finite element modeling}

FE simulations of the drop weight test and of the artificial athlete test were carried out with the commercial FE code Abaqus 6.11 (Simulia) [23]. 
Taking advantage of the axisymmetric geometry and loading conditions, only one half of the cross-

section of both test setups was modeled and discretized; sketches of the meshes are reported in Figures 3 and 4 for the drop weight test and the $F R$ test, respectively. To simplify the models and avoid (or limit) convergence problems due to the propagation of the impact-induced stress waves, the steel parts of the test setups (the striker and the clamping plates in Figures 3, all the plates along the load line of the artificial athlete in Figure 4) were considered as rigid bodies. The spring and load cell of the artificial athlete were instead modeled as elastic springs, with masses of $1.460 \mathrm{~kg}$ and $0.518 \mathrm{~kg}$ and stiffnesses of $2 \mathrm{kN} / \mathrm{mm}$ and $1 \mathrm{MN} / \mathrm{mm}$ respectively, as specified by the instrument manufacturer. Accordingly, only the specimen, the back plate in the drop weight test and the substrate in the $F R$ test were modeled as deformable continua. They were discretized using the fournode bilinear elements CAX4RH featuring reduced integration and hourglass control, implemented within a mixed (or hybrid) formulation and handling a piece-wise constant pressure, see [23]. The adopted meshes, depicted in Figures 3 and 4, turned out to be a good compromise between accuracy (especially as for wave reflection/propagation across the contact surfaces) and computational costs. The characteristic size of the meshing elements was $1 \mathrm{~mm}$ for the track samples, $0.2 \mathrm{~mm}$ for the HDPE back plate in the drop weight test and $2.5 \mathrm{~mm}$ for the substrate in the $F R$ test, respectively; this choice allowed attaining accurate, mesh-independent results also in terms of $F R$ values. The unilateral contact between the facing surfaces of all the bodies was enforced using a penalty method, to enhance convergence in the handled multi-body simulations. The friction coefficient at the sample-HDPE and the sample-steel contact surfaces was determined experimentally: it is $\mu_{\mathrm{A} \text { - }}$ HDPE $=0.68$ and $\mu_{\mathrm{A}-\text { Steel }}=0.98$, respectively.

The boundary conditions were enforced upon the clamping device of the drop weight tester (providing two self-equilibrated overall vertical forces $F_{c}$ ) and by constraining to zero the displacements along the lateral boundary of the substrate in the $F R$ test. As for this latter condition, the radius of the modeled region $(150 \mathrm{~mm}$, as indicated in Figure 4) was tuned so as to avoid that spurious stress wave reflections along the lateral surface may affect the time evolution of the stress 
state across the test specimen. The initial conditions were instead defined by setting everything at rest at the initial instant $t=0$ with exception of the striker (part 2 in Figures 3 and 4) featuring a vertical, downward velocity of $1.8 \mathrm{~m} / \mathrm{s}$ and $1.04 \mathrm{~m} / \mathrm{s}$ in the drop weight and $F R$ test, respectively. The solution of the nonlinear equations of motion was advanced in time adopting the HHT $\alpha-$ method [24], which is an enhanced version of the standard Newmark algorithm, able to damp spurious high-frequency oscillations in the solution linked to local deformation modes at element (or element patch) level. In this regard, it is worth noting that Abaqus automatically tailors the algorithmic coefficients to avoid excessive dissipation of the stored elastic energy, see [23]. As for the constitutive description of the deforming materials, the model parameters of the hyperelastic models used are given in the forthcoming Section 5.1, where the results of quasi-static and dynamic testing are gathered. The HDPE back plate in the drop weight test and the concrete substrate in the $F R$ test were instead modeled as linear elastic bodies featuring the following properties: Young's modulus $E_{H D P E}=500 \mathrm{MPa}$, Poisson's ratio $v_{H D P E}=0.43$ and density $\rho_{H D P E}=934$ $\mathrm{kg} / \mathrm{m}^{3}$; Young's modulus $E_{\text {Concrete }}=30 \mathrm{GPa}$, Poisson's ratio $v_{\text {Concrete }}=0.15$ and density $\rho_{\text {Concrete }}=2300$ $\mathrm{kg} / \mathrm{m}^{3}[25]$.

\section{Results and discussion}

\subsection{Experimental characterization and parameter identification}

Figure 5 shows an example of the stress-stretch curves recorded for materials A and NR under uniaxial compression, at a few low stretch-rates, $\dot{\lambda}$. Both materials show a slight dependence on stretch rate, with a higher stress for higher stretch rates. Hence, the materials' behavior is not really hyperelastic but rather weakly viscoelastic, as already observed in [17].

A quantitative assessment of the stretch rate dependence is obtained by evaluating the rate sensitivity index, $m$, conventionally defined as $m=[\partial \ln (\sigma) / \partial \ln (\dot{\lambda})]_{\lambda}[21,22]$ and approximated here as $\bar{m}=[\Delta \ln (\sigma) / \Delta \ln (\dot{\lambda})]_{\lambda}$ where the bar denotes averaging over pairs of stretch-rates tested, 
at constant stretch, $\lambda$. The stretch-rate sensitivity index so obtained, $\bar{m}$, is plotted in Figure 6 as a function of the stretch $\lambda$, for the two materials. Clearly both the absolute value of $\bar{m}$ and its dependence on stretch $\lambda$ are small in the ranges of stretches and stretch-rates of interest here.

The former result is coherent with the modest degree of viscoelasticity observed for these same materials in [17] as evidenced by the small magnitude of the loss factor $\tan \delta$ (around 0.1) measured in the small deformation region, where the stress-stretch behavior is linear. Although in the present work the characterization is extended to significantly larger deformations (namely: stretch ratios up to 0.2 for material A and 0.5 for material NR) where the behavior appears quite non-linear (Figure 5), still nearly the same, limited rate sensitivity is found. This result corroborates the choice of rate independent models for the purposes of the present FE numerical analysis.

Nevertheless, for the identification of the parameters of the hyperelastic models the modest rate dependence shown by the experimental data was taken into account, for sake of accuracy: the values of the parameters were determined for a stretch rate of $60 \mathrm{~s}^{-1}$, which is typical of $F R$ tests, as estimated from the time- and length- scales typical of the impact event undergone by the track surfaces in the $F R$ test and validated a posteriori by the simulations analysis. To that purpose, values of the parameters at low rates were first obtained via best fitting of Eqs. (2) and (3) to the experimental stress-stretch data reported in Figure 5; these values were subsequently extrapolated to $60 \mathrm{~s}^{-1}$ using a simple linear fit of said parameters as a function of the logarithmic stretch rate, in view of the modest rate dependence remarked above.

Figure 7 shows, just as an example, the fitting of the experimental stress-stretch data at one stretch rate with both the Neo-Hookean and the Mooney-Rivlin's constitutive equations, Eqs. (2) and (3) respectively. In the case of material A (Figure 7a), the Mooney-Rivlin's constitutive law fits the experimental data somewhat better than the Neo-Hookean model over the entire stretch range explored; in the case of NR (Figure 7b), the two constitutive equations appear equivalently good over the stretch range covered here. To keep symmetry between the two illustrative materials 
considered in this study (track and reference), the Mooney-Rivlin's constitutive equation was used for both materials in the numerical modeling.

The values of Mooney-Rivlin's coefficients $C_{10}$ and $C_{01}$ obtained from the least square fitting are reported in Figure 8a and 8b for material A and NR, respectively, as a function of stretch rate. Even though the data points are quite scattered, the simple linear fit (in a logarithmic stretch rate scale) used to interpolate them and obtain the extrapolated value at $60 \mathrm{~s}^{-1}$ appears sufficiently accurate as indicated by the $95 \%$ prediction limits, also shown in Figure 8 . An exception was made for the NR's parameter $C_{10}$, which showed no clear dependence on stretch rate and was thus taken as a rate-independent average.

The resulting model parameters for both materials are summarized in Table 1.

\subsection{Drop weight tests and simulations}

Before modeling the FR test, a preliminary comparison between experimental data and simulated results for the case of the drop weight test was carried out, aiming at validating the numerical method chosen, the assumption of modeling the material as homogenous and the extrapolation of the constitutive parameters from quasi-static to high stretch rates. Moreover, in view of the significant uncertainty in the Mooney-Rivlin's coefficients $C_{10}$ and $C_{01}$ due to the large degree of extrapolation, it allowed to check the sensitivity to a change in their values. This comparison was performed only on material A, as its case was deemed more critical with respect to homogeneity and rate dependence. The main outcome of this analysis is reported in Figure 9, which displays the force vs. time evolution during the test. The overall prediction is quite accurate, and the peak force is overestimated by only $3 \%$. To assess the sensitivity of the analysis to the values of the parameters, Figure 9 shows also the results of additional simulations in which one of the two parameters, either $C_{10}$ or $C_{01}$, was held fixed at the extrapolated value, while the other one was assigned either the lower or the upper $95 \%$ confidence value. It can be observed that such variations 
have only a minor effect: the peak force is overestimated by $8 \%$ in the worst case. The validity of

\subsection{Force Reduction tests and simulations}

The results of numerical simulations and experimental results of a $F R$ test are compared in Figure 10, for a $16 \mathrm{~mm}$ thick sample of material A (Figure 10a) and NR (Figure 10b). The overall trend of the force is well described by the simulation, and the relative error in peak force prediction is less than $1 \%$ for material A and about $3 \%$ for NR.

The simulated force-time curve for material A displays some high-frequency fluctuations, which are not observed in the experimental record, perhaps because of the low acquisition frequency of the experimental apparatus used. A closer analysis of the simulation output suggests that these features are related to the unilateral contact between the artificial athlete base plate and the load cell. Figure 10a shows also the predicted evolution of the relative displacement between the base plate and the load cell of the artificial athlete during impact (right y-axis). Positive values of this relative displacement correspond to separation between the two parts, whereas zero or negative values are representative of contact occurring between parts (actually, negative values would represent penetration, allowed by the penalty algorithm adopted). It can be observed that force oscillations are mainly associated to transitions from contact to separation, or vice-versa; as such, they can be considered as (minor) numerical artifacts.

To check the capability of the numerical model to predict the force evolution also on varying sample thickness, a test was carried out on a $64 \mathrm{~mm}$ thick specimen of material A (Figure 11). The simulation matches very well the experimental data both in terms of the full force-time history (and hence in terms of evolution of the elastic energy stored in the track material) and in terms of maximum force value (determining the $F R$ index) which exhibits a relative error of only $2 \%$. 


\subsection{Data analysis}

For the evaluation of $F R$ according to its definition (eq. 1) both the experimental and the simulated force-time curves were processed with a filter having a $9^{\text {th }}$ order Butterworth characteristic with a -3 dB frequency of $120 \mathrm{~Hz}$, as specified in the standard [12], before determining $F_{\max }$ from each of the experimental or simulated test. The reference force value, $F_{\text {max,ref, }}$ was also determined, experimentally on concrete and numerically from a simulated $F R$ test with the base plate resting directly on the substrate. The two values agreed quite well.

The results of this elaboration for the two materials, material A and NR, with varying sample thickness are shown in Figure 12: again a very good agreement between experiments and simulations is observed over the whole thickness range covered, with Root Mean Square Errors between experimental and predicted $F R$ values of $1.44 \%$ and $1.22 \%$ for material A and NR, respectively.

Figure 12 also confirms the experimental results reported in [17]: $F R$ increases with thickness and tends to level off to an asymptotic value, $F R_{\infty}$, which is nearly reached at a surface thickness, $s$, of about $120 \mathrm{~mm} . F R_{\infty}$ appears to be an intrinsic (geometry independent) material property. It is thus interesting to seek its correlation with the fundamental mechanical properties of the material, as done in [17]. In the present case, the stress-stretch behavior of the two materials is non-linear. For that a Young's modulus, estimated from the Mooney-Rivlin's parameters as $E=2 \times 3 \times\left(C_{10}+C_{01}\right)$, was chosen as representative of material's stiffness and a series of simulations were run on several "virtual" track surfaces, characterized by different values of their Mooney-Rivlin's parameters and having a thickness of $120 \mathrm{~mm}$. The $F R_{120}$ values so obtained are plotted in Figure 13 versus the relevant Young's modulus. An inverse dependence can be observed, similar to the one found in [17] for the experimental $F R_{\infty}$ vs the material's dynamic rigidity, $\left|E^{*}\right|=\sqrt{E^{\prime 2}+E^{\prime 2}}$ (where $E^{\prime}$ and $E^{\prime \prime}$ are the conservative and dissipative components of the dynamic modulus, $E^{*}$ ) measured at $10 \mathrm{~Hz}$. 
The excellent predictive capability of the FE model based on purely elastic constitutive behavior demonstrated in the present work along with the strict correlation between $F R_{120} \approx F R_{\infty}$ and Young's modulus displayed in Figure 13 strongly enforce the idea that $F R_{\infty}$ depends essentially on the elastic component of the viscoelastic response of the material. Yet, a definitive conclusion as to this regard could be reached only by testing materials having a stronger viscoelastic character than the ones examined in the previous and in the present papers.

Finally, the good correlation displayed in Figure 13 between $F R$ and the stiffness of the material as expressed by the Young's modulus may question the need of taking materials non-linearity into account. Indeed, from the numerical simulations carried out in the present study it can be estimated a maximum stretch reached in the artificial athlete test of around 0.9: for this deformation the degree of non-linearity of the materials examined here is limited to about $10 \%$ in the case of material A and $13 \%$ in the case of NR. The opportunity of adopting a linear elastic constitutive equation to predict FR in place of the hyperelastic one, as well as the influence of larger degrees of non-linearity would require further investigation.

\section{Conclusions}

In this work a finite element model of the Force Reduction test was developed. The athletics track materials' behavior was modeled by the hyperelastic Mooney-Rivlin's constitutive equation, whose parameters where obtained from quasi-static compression tests. As the materials response turned out to be (moderately) rate dependent, a compromise procedure was used: the values of the model parameters at the relevant rates were determined by extrapolation from lower rates and then assumed as constants in the numerical simulation. This procedure was demonstrated to be valid even for large extrapolation by comparing simulations and experiments from a drop weight test. The predictive capability of the numerical model proved to be excellent both in terms of the forcetime response in an artificial athlete test and the derived $F R$ value. This model can therefore be exploited both for materials selection and optimal design of new surfaces for specific applications 
or even to predict other characteristics of the track, such as, for example, its "Vertical Deformation" [13].

Besides, the model showed itself fit for accurately describing the force reduction's dependence on track thickness also in the case of non-homogeneous materials: it confirmed that the value of force reduction increases with increasing thickness and tends to a limiting value, which is indeed related to the stiffness of the constituent material. The cushioning ability of athletics track surfaces as described by its $F R$ value appears to be essentially determined by the material's elastic response and by the system dynamics, rather than the intrinsically dissipative viscoelastic characteristics. Yet, the viscoelastic dissipation, especially at high frequencies, may still play an important role in protecting the athletes' health, something that cannot be fully grasped by Force Reduction alone.

The present study could also provide a reliable basis to develop more complex models to investigate the interaction between sport surface and athlete's shoe or foot. A detailed analysis of the whole impact phenomenon during the test performed with the artificial athlete - made possible by the developed simulation approach - is for sure widely more informative than a limited single value of FR. Coupled with biomechanical considerations (on the athlete's side) it may lead to deeper understanding of the material's role in improving the safety and efficiency of a track surface. 


\section{REFERENCES}

1. Silva RM, Rodrigues JL, Pinto VV, Ferreira MJ, Russo R, Pereira CM (2009) Evaluation of shock absorption properties of rubber materials regarding footwear applications. Polymer. Test. 28: 642-647

2. Schwellnus MP, Jordaan G, Noakes T (1990) Prevention of common overuse injuries by the use of shock absorbing insoles. A prospective study. Am. J. of Sports Med 18: 636-641

3. Voloshin A (1981) Influence of artificial shock absorbers on human gait. Clinical Orthopaedics and Related Research 160: 52-56

4. Farley C, Houdijk H, Strien CV, Louie M (1998) Mechanism of leg stiffness adjustment for hopping on surfaces of different stiffnesses. J. Appl. Phys. 85: 1044-1055.

5. Kerdok AE, Biewener AA, McMahon TA, Weyand PG, Herr HM (2002) Energetics and mechanics of human running on surfaces of different stiffnesses. J. Appl. Phys. 92: 469-478

6. MacMahon TA, Greene PR (1979) The influence of track compliance on running. J. of Biomech. 12:893-904

7. Brughelli M, Cronin J (2008) A review of research on the mechanical stiffness in running and jumping: methodology and implications. Scand. J. Med. Sci. Sports 18: 417-426.

8. Baroud G, Nigg BM, Stefanyshyn DJ (1999) Energy storage and return in sport surfaces. Sports Eng. 2: 173-180

9. Stefanyshyn DJ, Nigg BM In: Proceedings of the 3rd Symposium on Sports Surfaces, Calgary, 31-46 (2003).

10. Thomson RD, Birkbeck AE, Lucas TD (2001) Hyperelastic modelling of nonlinear running surfaces. Sports Eng. 4: 215-224

11. Aerts P, Clercq DD (1993 Deformation Characteristics of the heel region of the shod foot during a simulated heel strike: The effect of varying midsole hardness. J. Sports Sci. 11: $449-461$

12. IAAF Track Facilities Testing Protocols, (2009) 
13. IAAF Track and Field Manual, (2008)

14. EN 14808 Surfaces for sports areas - Determination of shock absorption

15. EN 14809 Surfaces for sports areas - Determination of vertical deformation

16. Durà JV, Garcìa AC, Solaz J (2002) Testing shock absorbing materials: the application of viscoelastic linear model. Sports Eng. 5: 9-14

17. Benanti M, Andena L, Briatico-Vangosa F, Pavan A (2013) Characterization of the viscoelastic behavior of athletics track surfaces in relation to their "force reduction" property. Polymer Testing 32: 52-59

18. Nigg BM, Yeadon MR (1987) Biomechanical aspects of playing surfaces. J Sports Sci. 5: $117-145$

19. Macosko CW, Rheology. Principles, measurements and applications, chapter 1, Wiley-VCH New York, (1994)

20. Ali A, Hosseini M, Sahari BB (2010) A review for constitutive models for rubber-like materials. Am. J. Eng. Appl. Sci. 3: 232-239

21. Goble DL, Wolff EG (1993) Strain-rate sensitivity index of thermoplastics. J. Mat. Sci. 28: $5986-5994$

22. Walley SM, Field JE (1994) Strain rate sensitivity of polymers in compression from low to high rates. DYMAT Journal 1: 211-227

23. Abaqus 6.11 User Manual. Dassault Systèmes: Vélizy-Villacoublay, France, 2011.

24. Hughes TJR. The Finite Element Method. Linear Static and Dynamic Finite Element Analysis. Dover, New York, 2000.

25. Cambridge Engineering Selector - Edupack - 2012 Version Granta Design Limited, Cambridge, UK, 2012. 


\section{Acknowledgements}

1

2

3

4

5

6

7

8

9

10

11

12

13

14

15

16

17

18

19

20

21

22

23

24

25

26

27

28

29

30

31

32

33

34

35

36

37

38

39

40

41

42

43

44

45

46

47

48

49

50

51

52

53

54

55

56

57

58

59

60

61

62

63

64

65

The authors wish to thank Mr. Paolo Dalboni for providing the workstation that has been used for performing numerical simulations. 


\section{FIGURES' AND TABLE CAPTIONS}

Fig. 1 Artificial athlete apparatus. 1 falling mass; 2 electromagnetic brake; 3 load cell; 4 base plate; 5 upper plate; 6 spring; 7 track surface; 8 substrate

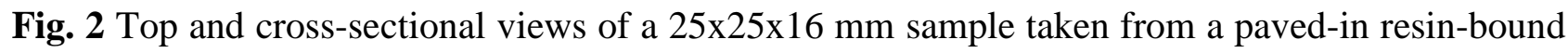
EPDM-based running track surface

Fig. 3 Sketch of the FE model of the drop weight tower test: 1 -axis of symmetry, 2 - striker, 3 clamping plates, 4 - material A specimen, 5 - HDPE substrate

Fig. 4 Sketch of the FE model of the FR test: 1 - axis of symmetry, 2 - falling body, 3 - upper plate, 4 - spring, 5 - load cell, 6 - base plate, 7 - test specimen, 8 - substrate

Fig. 6 Stretch-rate sensitivity index, $\bar{m}$, as a function of stretch, $\lambda$, averaged over pairs of experimental stress-stretch curves from compression tests as from Figure 5: (a) material A, and (b) NR

Fig. 7 Comparison of experimental nominal stress - stretch data in compression tests (points) and best fitting (lines) according to Neo-Hookean (solid line) and Mooney-Rivlin's (dashed line) constitutive equations: (a) material A, (b) NR. The coefficient of determination $\left(\mathrm{R}^{2}\right)$ for each fitting is also reported in the corresponding legend

Fig. 8 Mooney-Rivlin's coefficients (a) $C_{10}$ and (b) $C_{01}$, obtained by best fitting of the compression test data at varying stretch rate (symbols - material A: squares; NR: circles). Interpolation of the stretch rate dependence by a semi-logarithmic (linear-log) fitting (solid lines), and relevant 95\% prediction interval (dashed and dashed-dotted lines)

Fig. 9 Comparison between experimental and simulated force-time curves in a drop weight test performed on material A, and sensitivity to variations in parameters (a) $C_{10}$ and (b) $C_{01}$ Fig. 10 Comparison between $F R$ experiments and simulations, performed on $16 \mathrm{~mm}$ thick samples of (a) material A and (b) NR. The right y-axis of figure (a) reports the simulated relative displacement between the test foot and the load cell during the test 
Fig. 11 Comparison between $F R$ experiment and simulation, performed on a $64 \mathrm{~mm}$ thick sample of material A

Fig. 12 Dependence of $F R$ on track surface's thickness for the two materials. Comparison of experimental (filled points) and simulated (hollow points) results. Each point represents a single experiment or simulation. Lines are drawn just as a visual aid

Fig. 13 Force Reduction for a surface thickness of $120 \mathrm{~mm}, F R_{120}$, versus Young's Modulus calculated as $E=2 \times 3 \times\left(C_{10}+C_{01}\right)$. Diamonds corresponds to simulations performed on material A and NR, while squares are from simulations performed on different "virtual" track surfaces, characterized by the values of Mooney-Rivlin's parameters $C_{10}$ and $C_{01}$ indicated in parentheses. Dotted line: drawn by guesswork. For the sake of comparison the force reduction asymptotic values, $F R_{\infty}$, experimentally determined in [17] for material A and NR are also reported, here indicated by horizontal dashed lines

Table 1 Mooney-Rivlin's coefficients at stretch rate $60 \mathrm{~s}^{-1}$ (extrapolated values) 
Figure 1

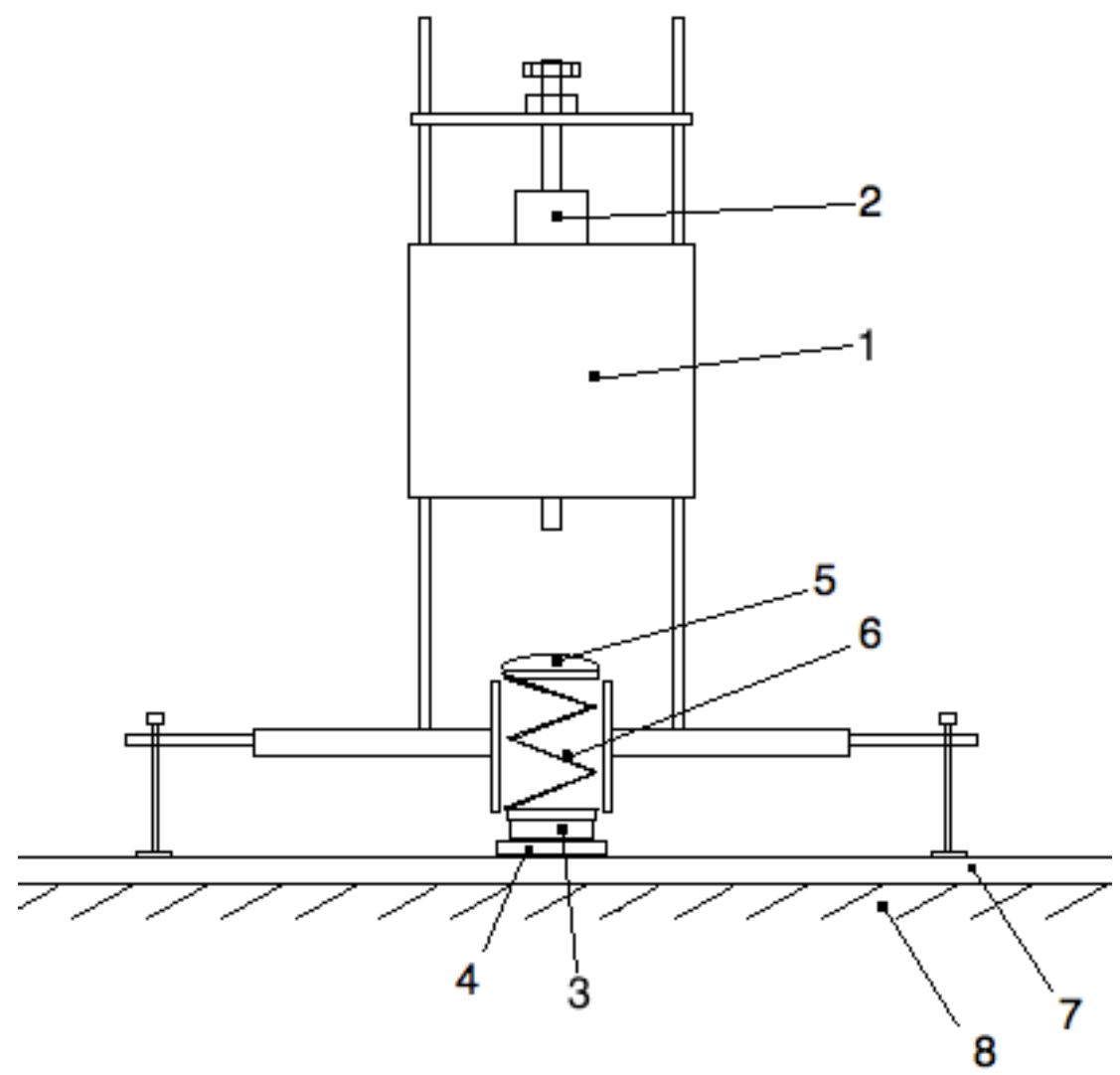

Fig. 1 
Figure 2
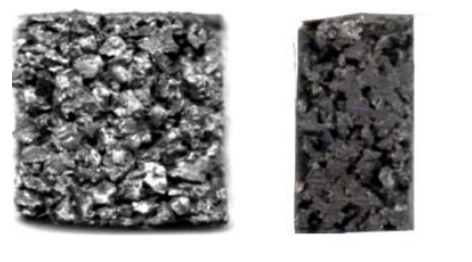

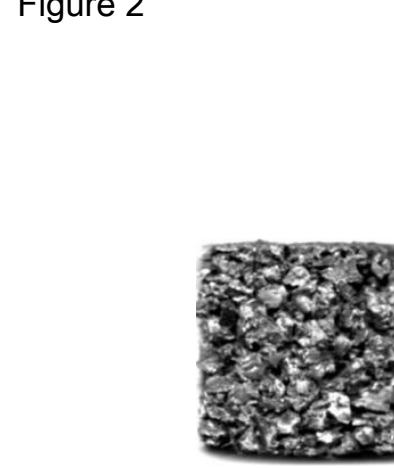

2

.

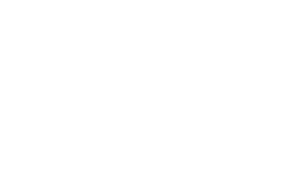

(1)

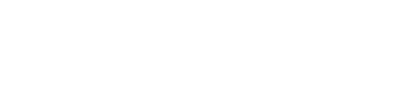

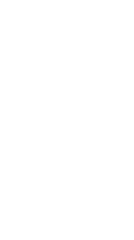

(2)
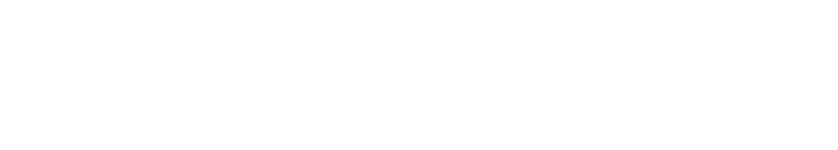

\footnotetext{
Fig. 2

Fig. 2
} 


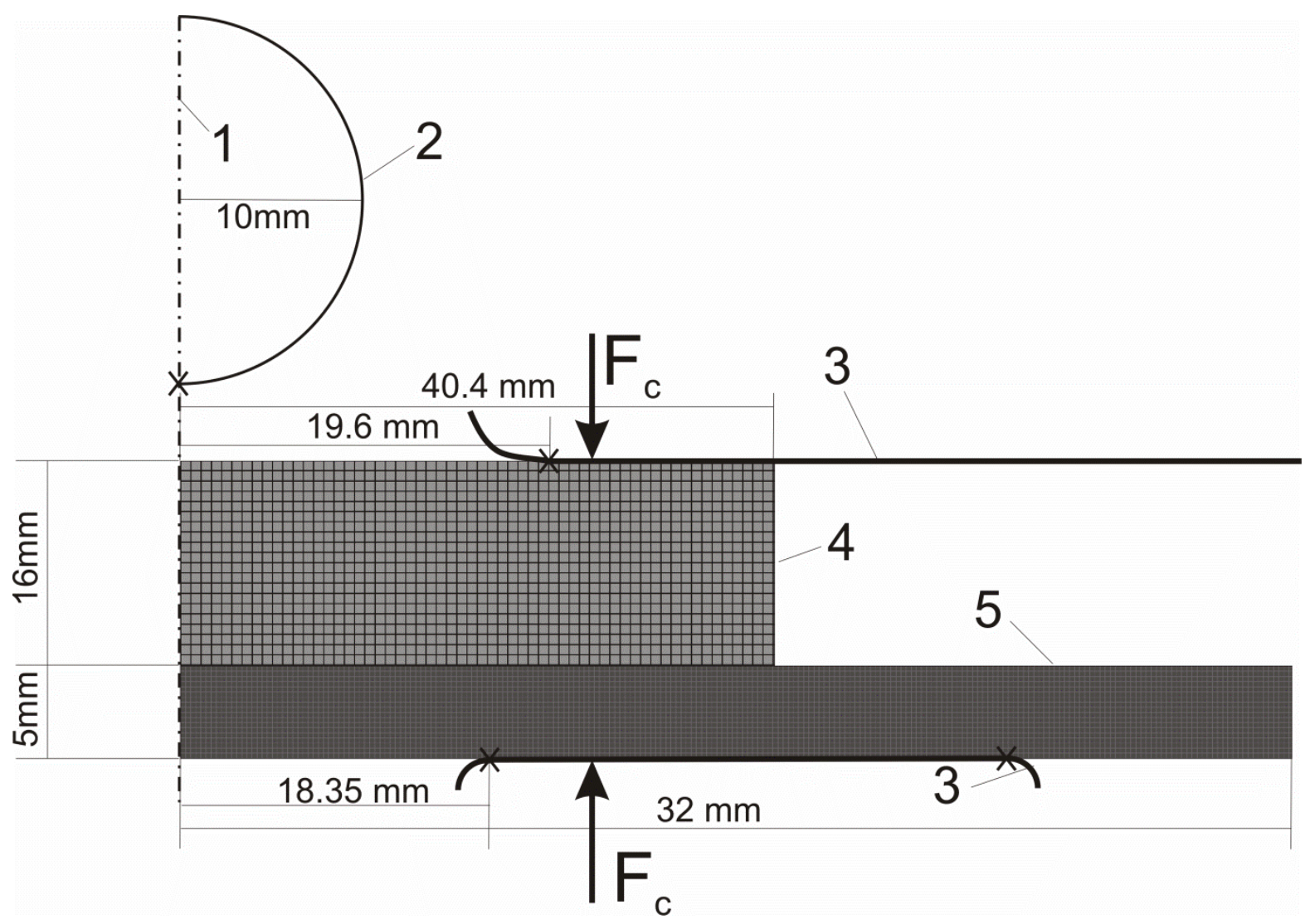

Fig. 3 


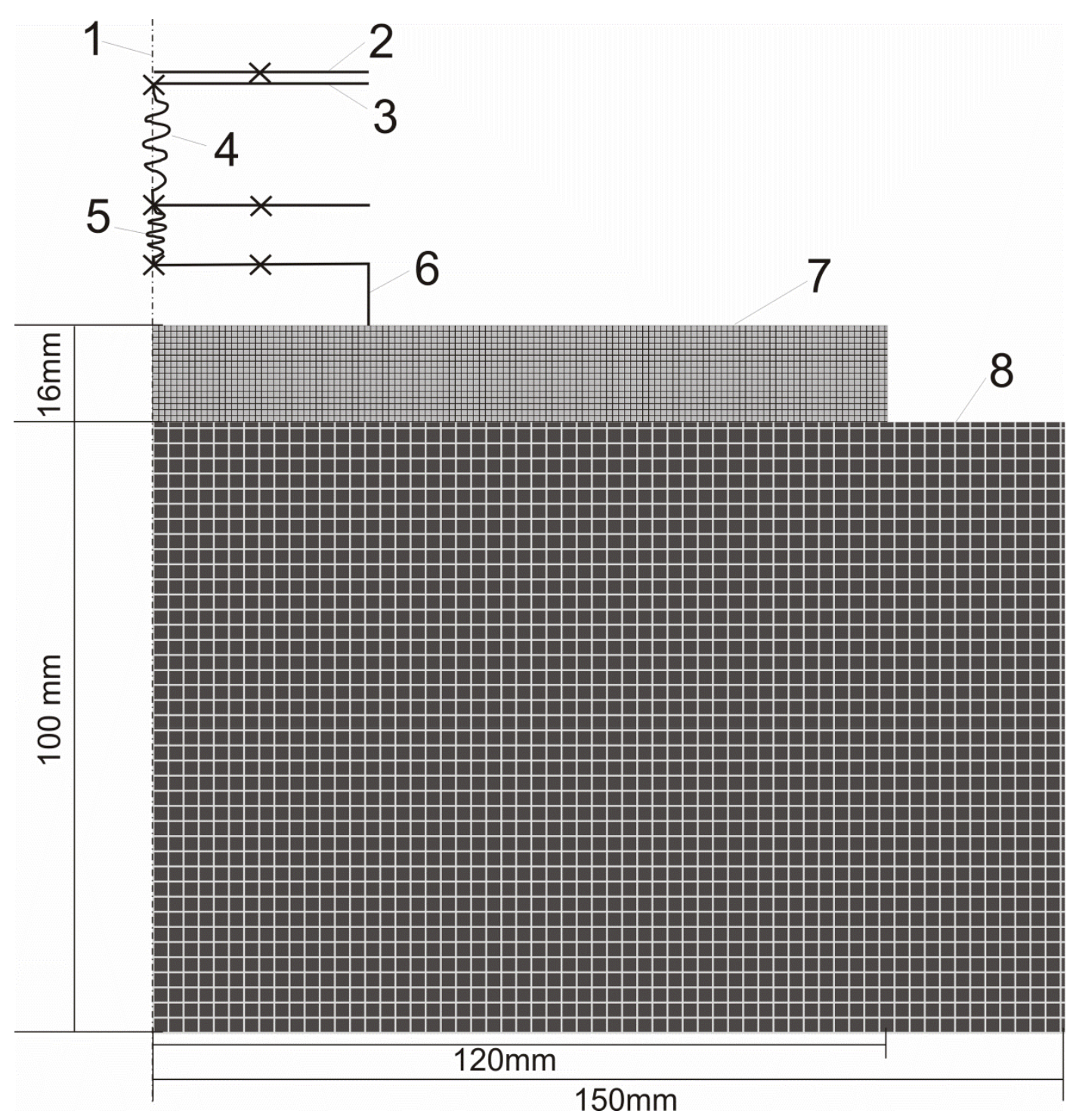

Fig. 4 
Stretch, $\lambda[-]$

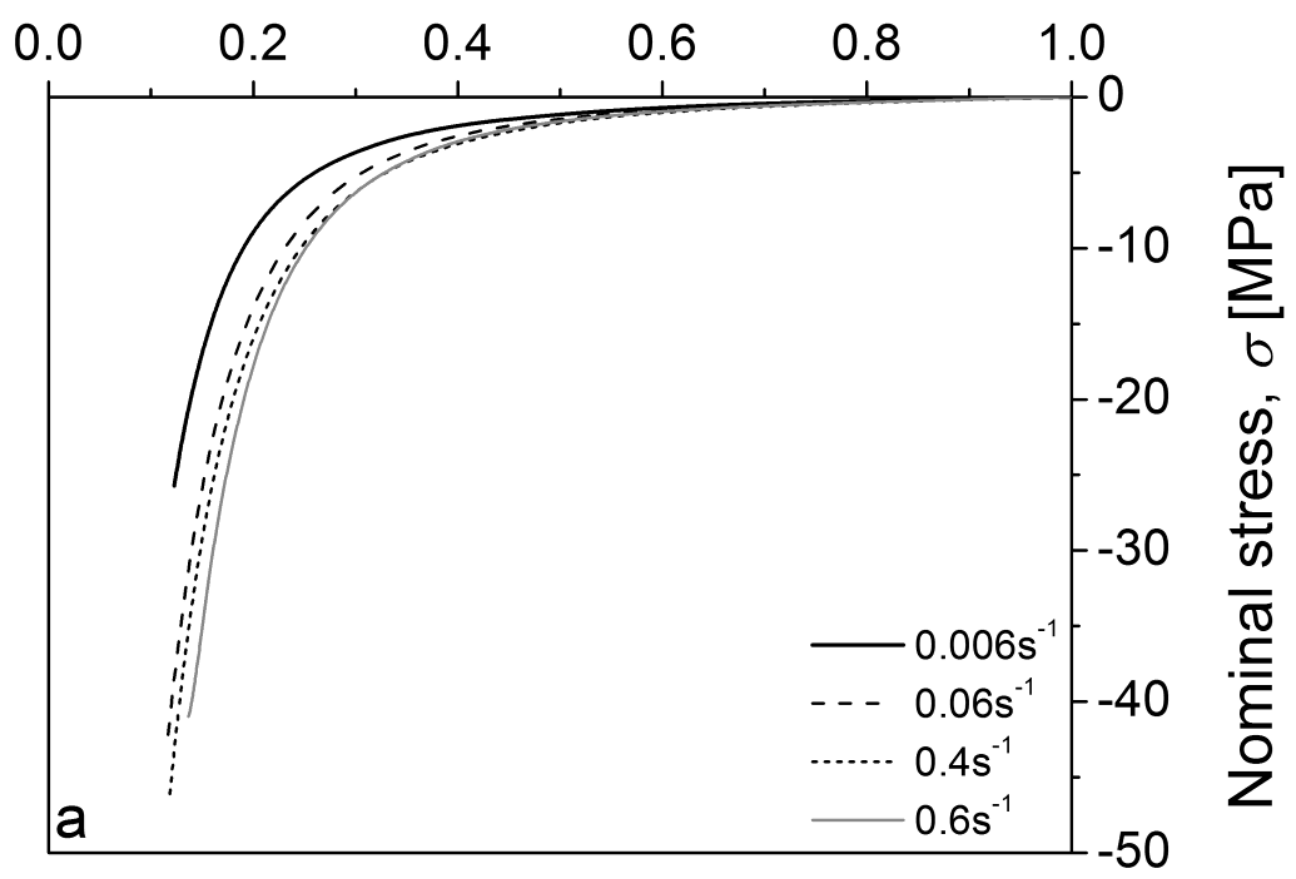

Fig. $5 a$ 


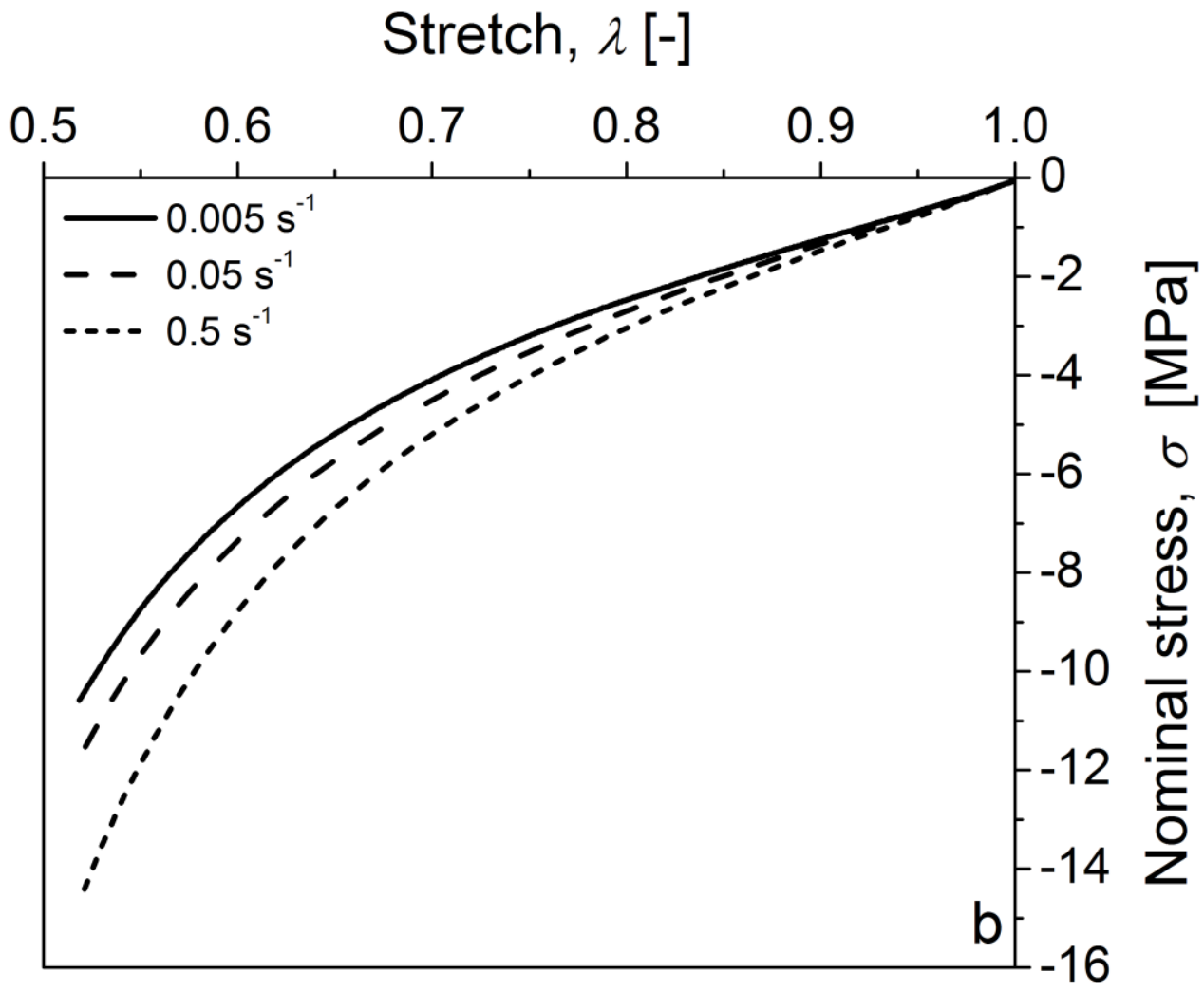

Fig. 5b 


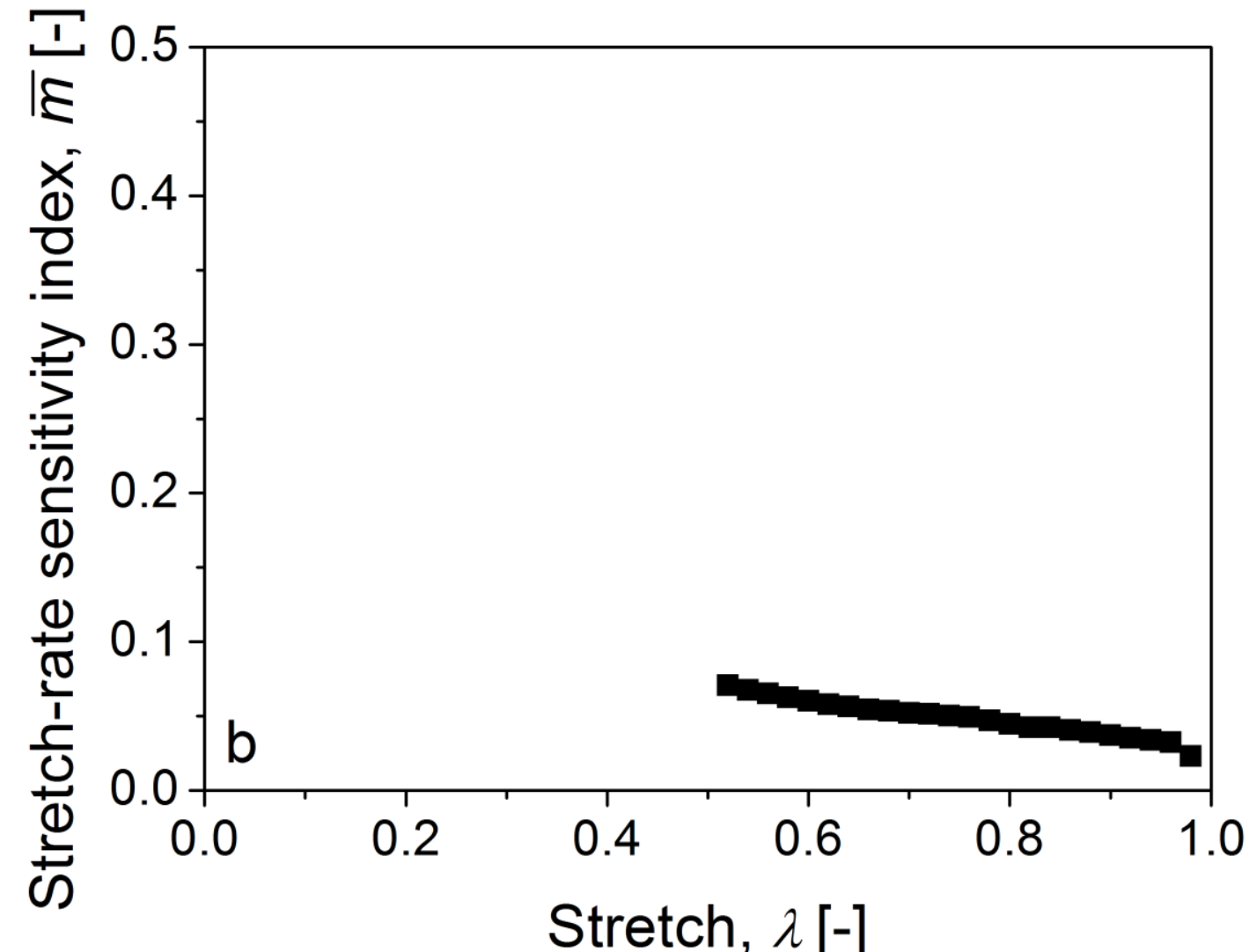

Fig. 6b 


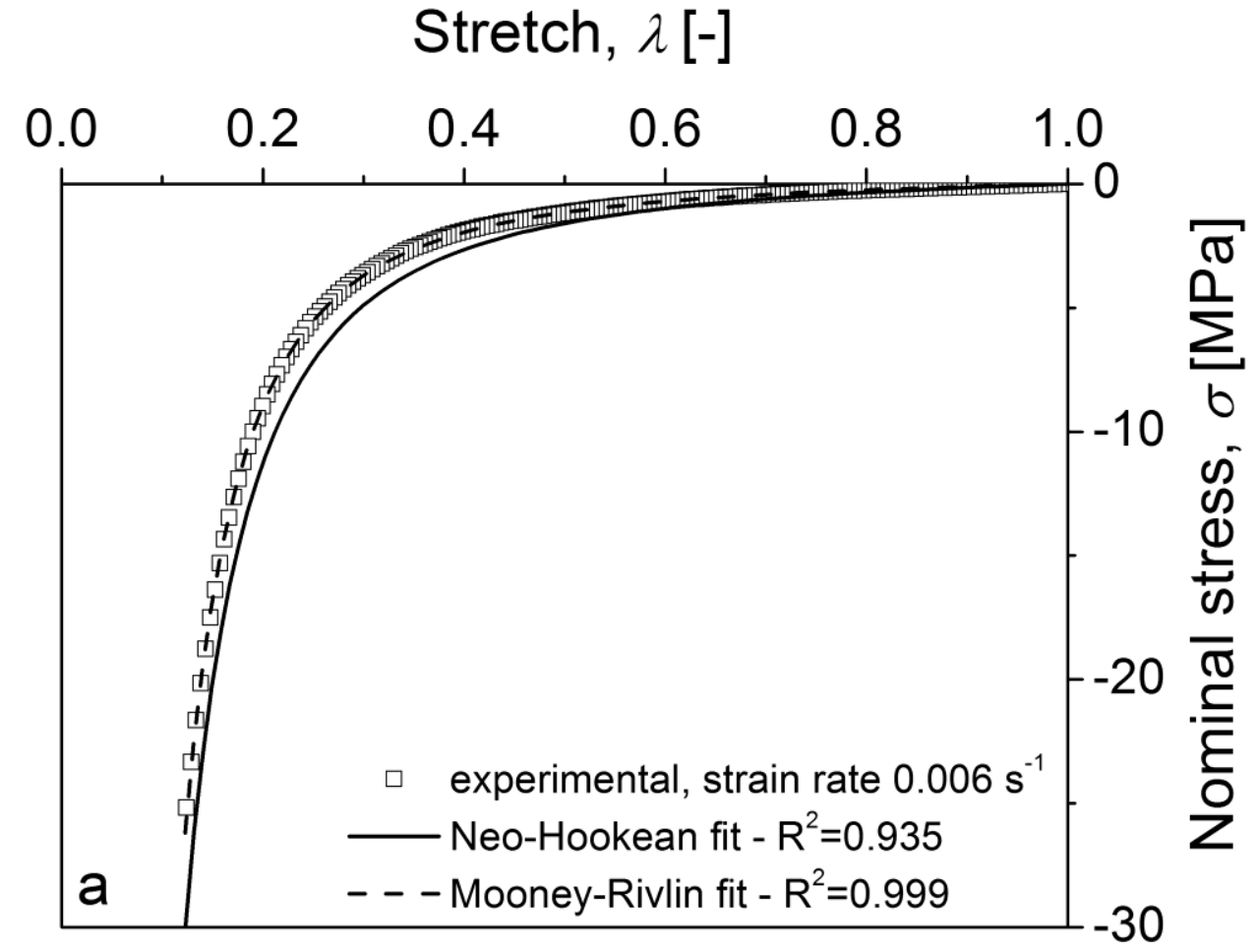

Fig. 7a 


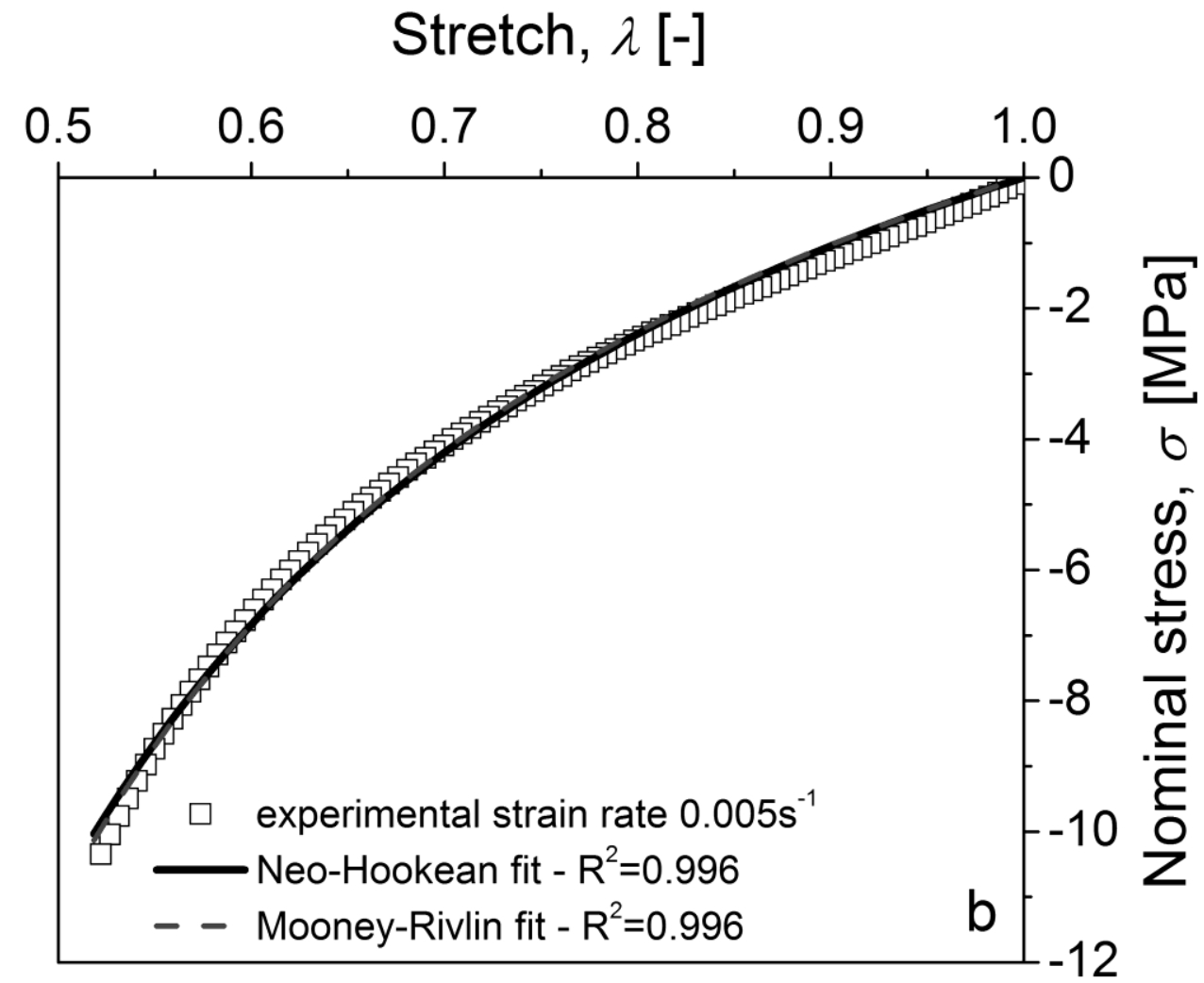

Figure 7b 


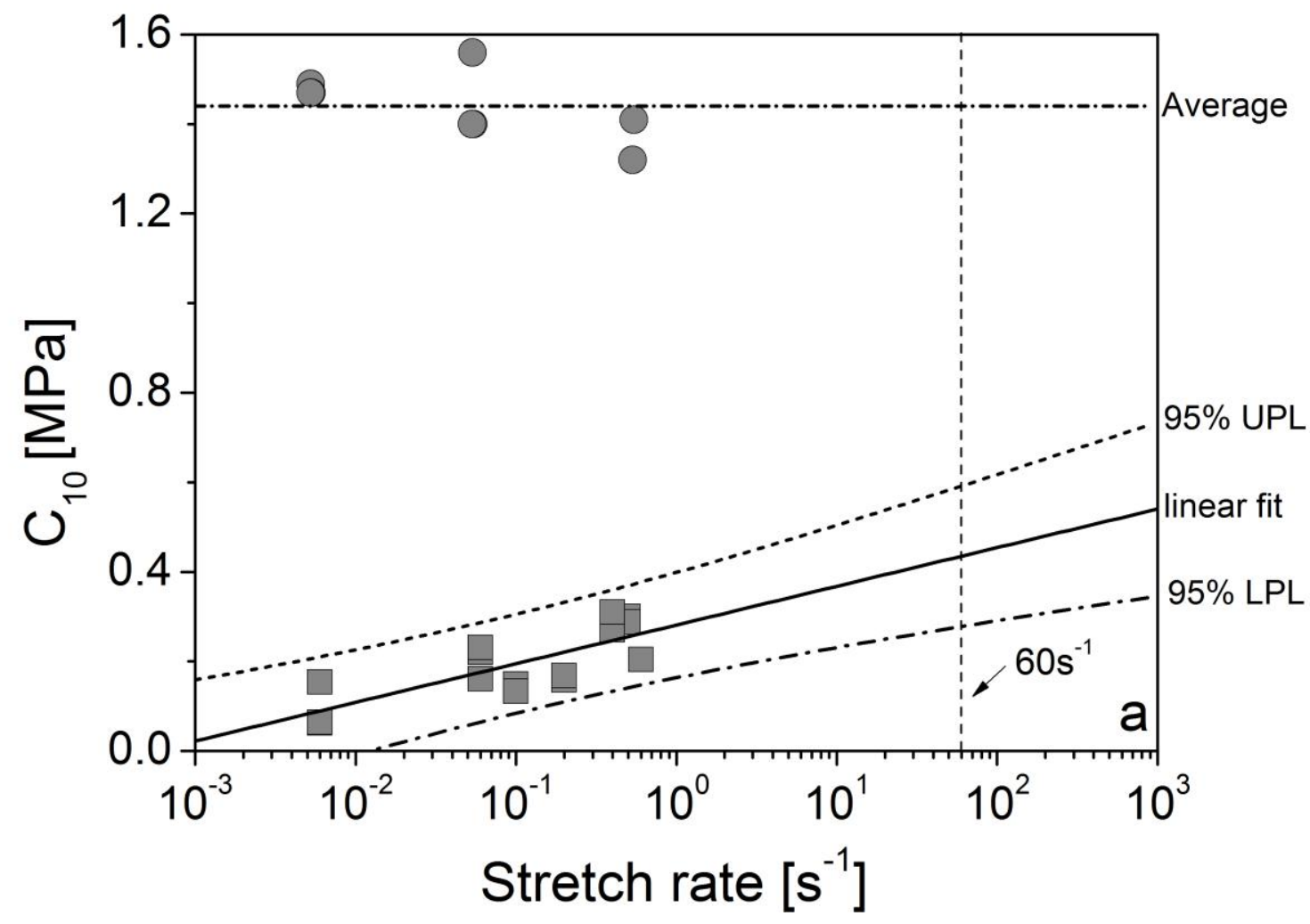

Fig. 8a 


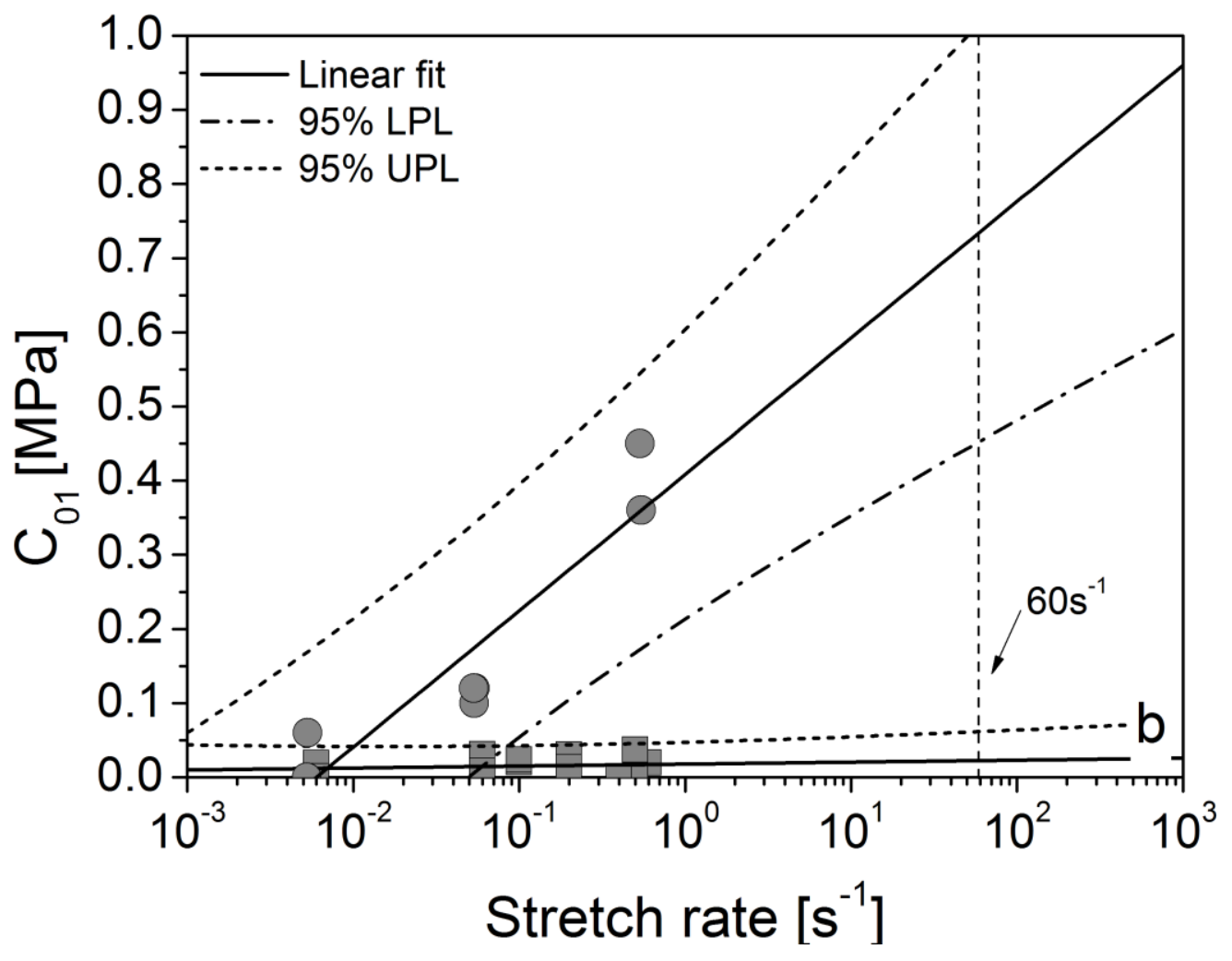

Fig. 8b 


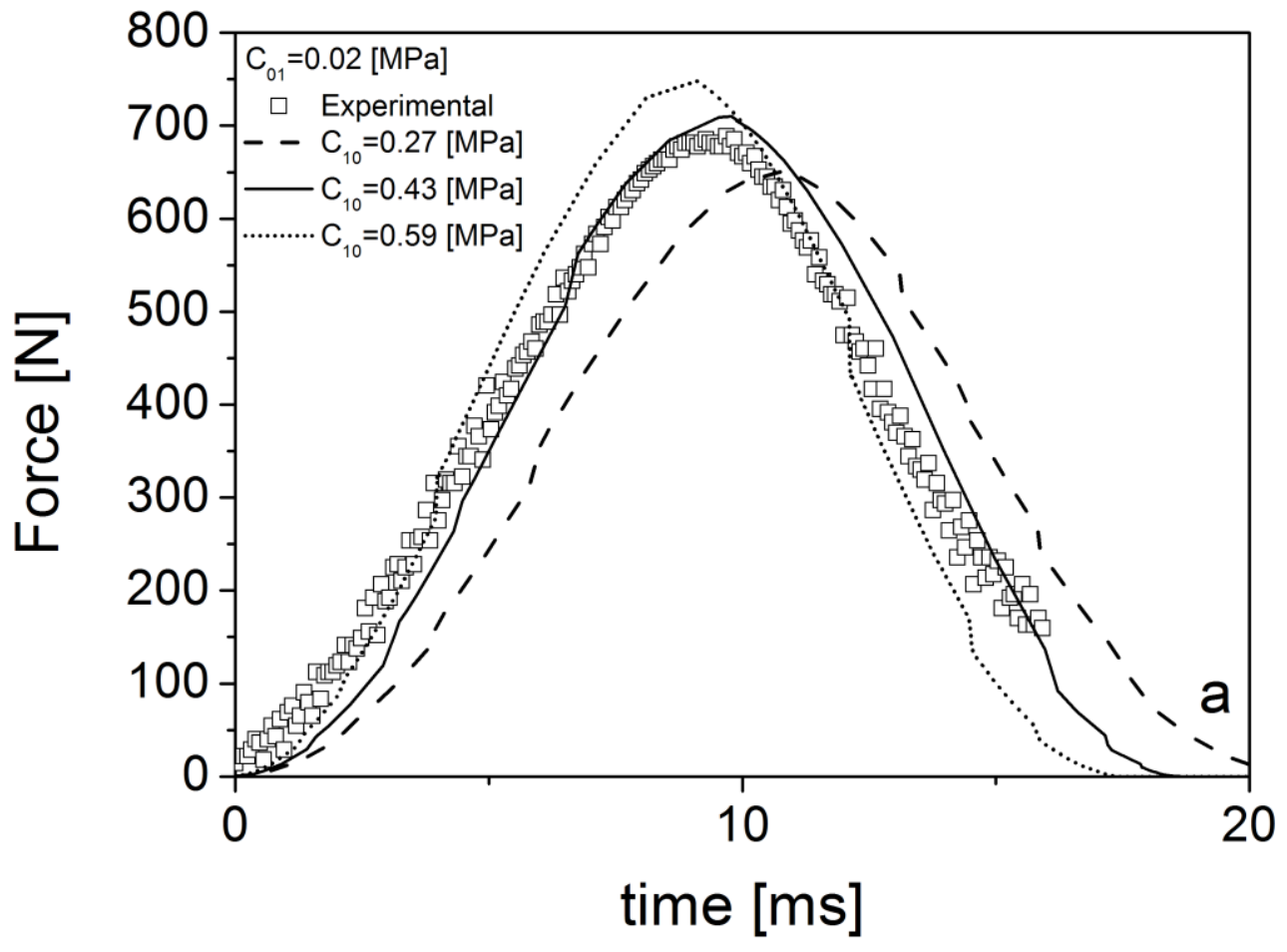

Fig. 9a 


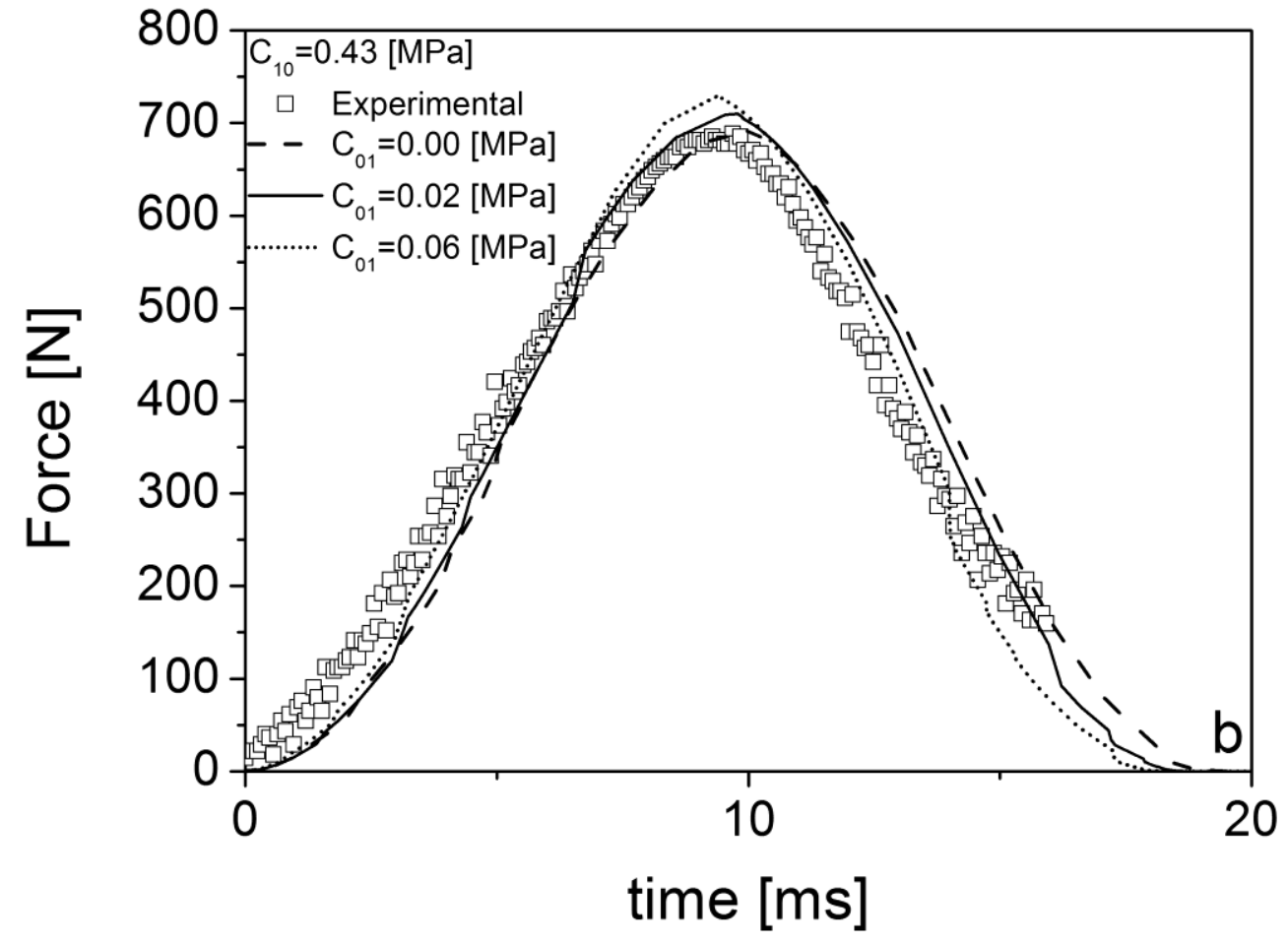

Fig. 9b 


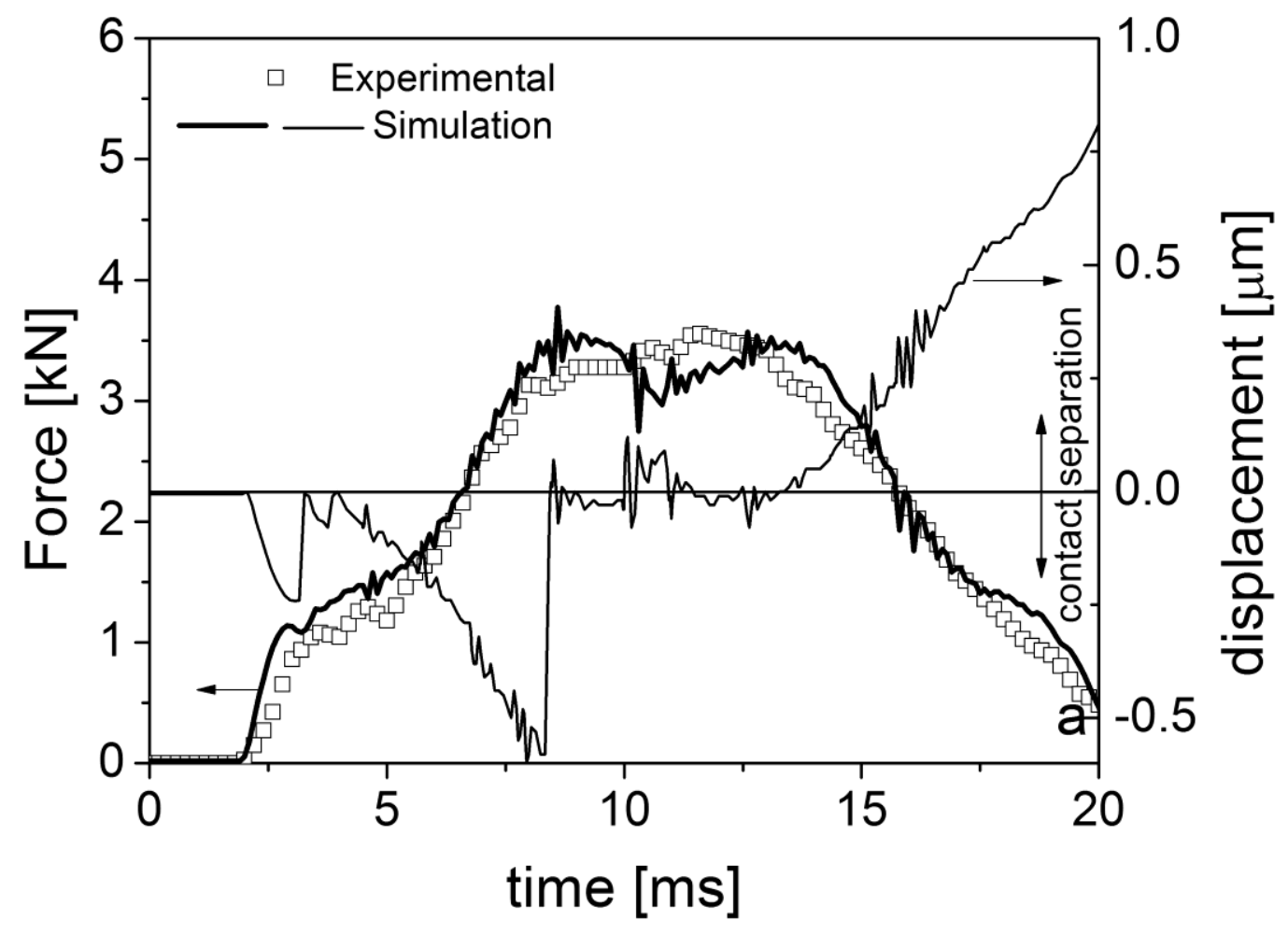

Fig. 10a 


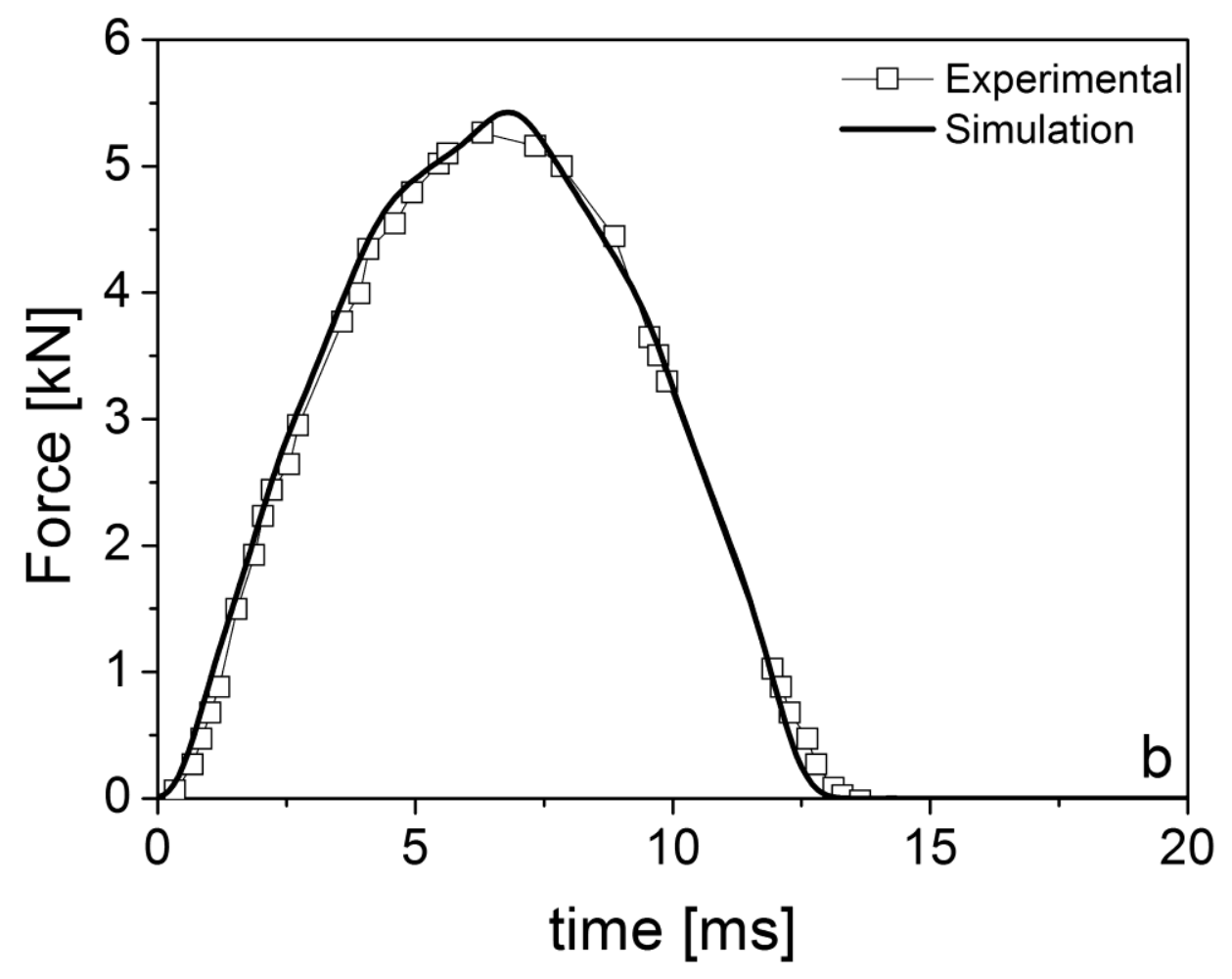

Fig. 10b 


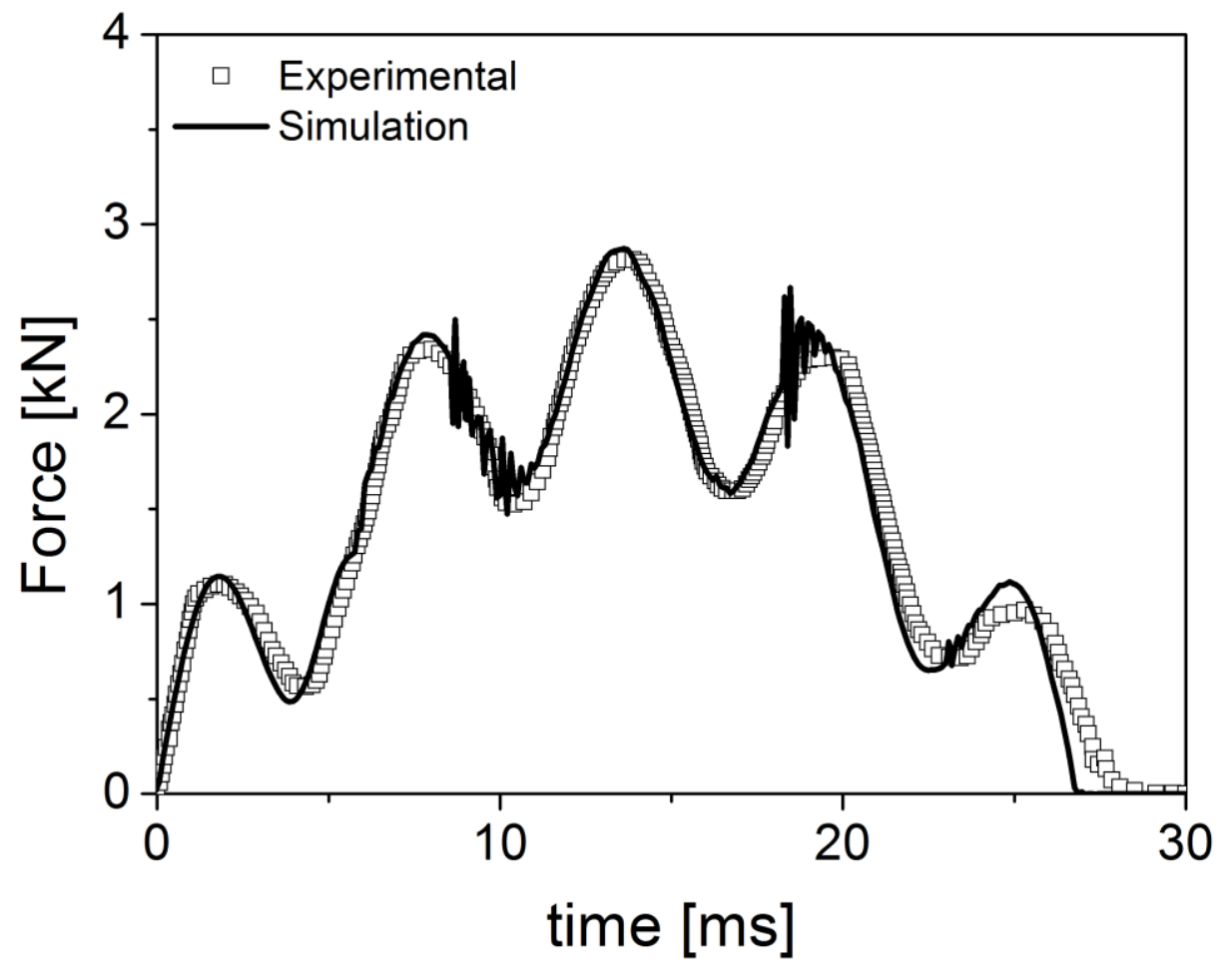

Fig. 11 


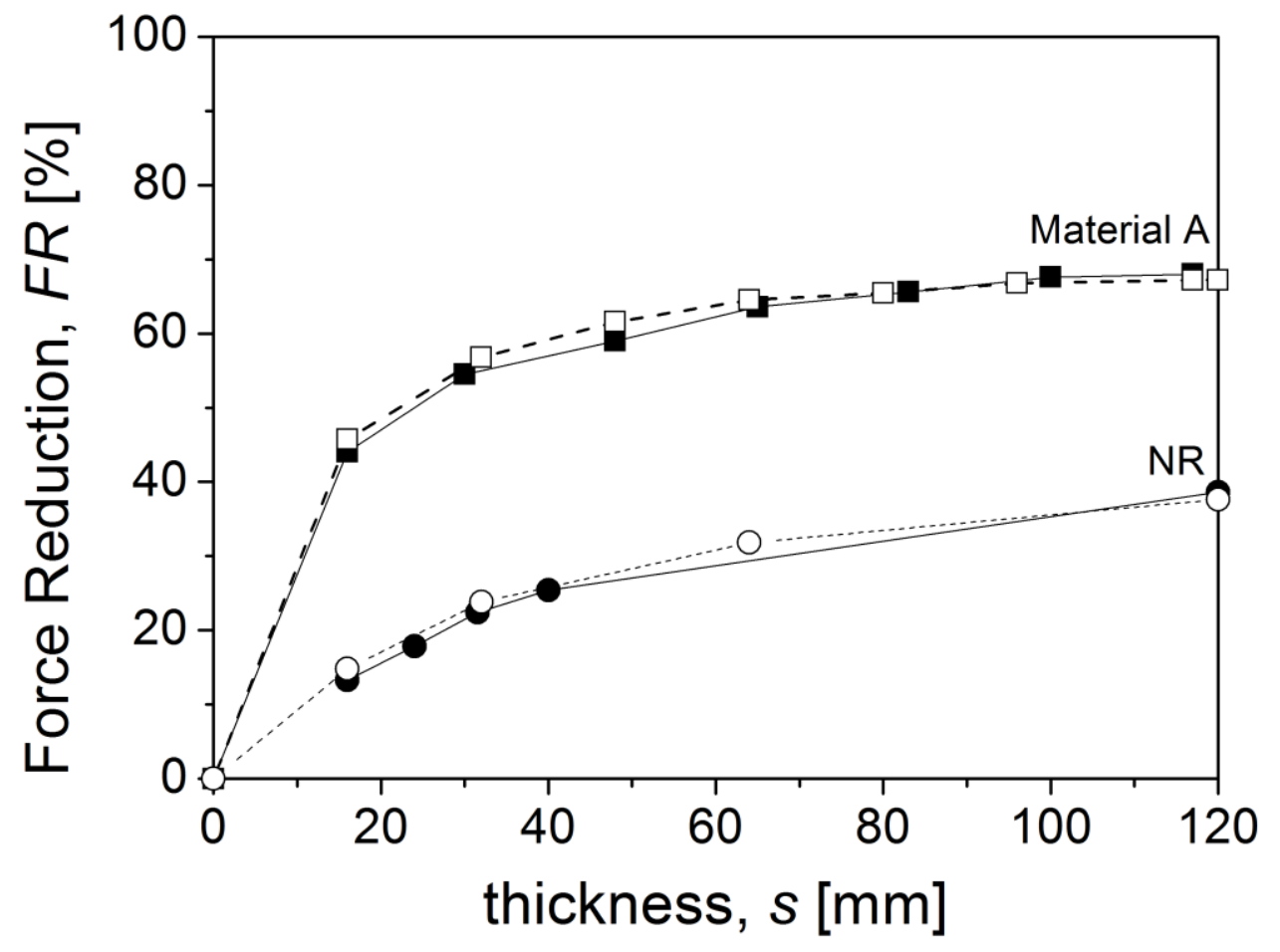

Fig. 12 


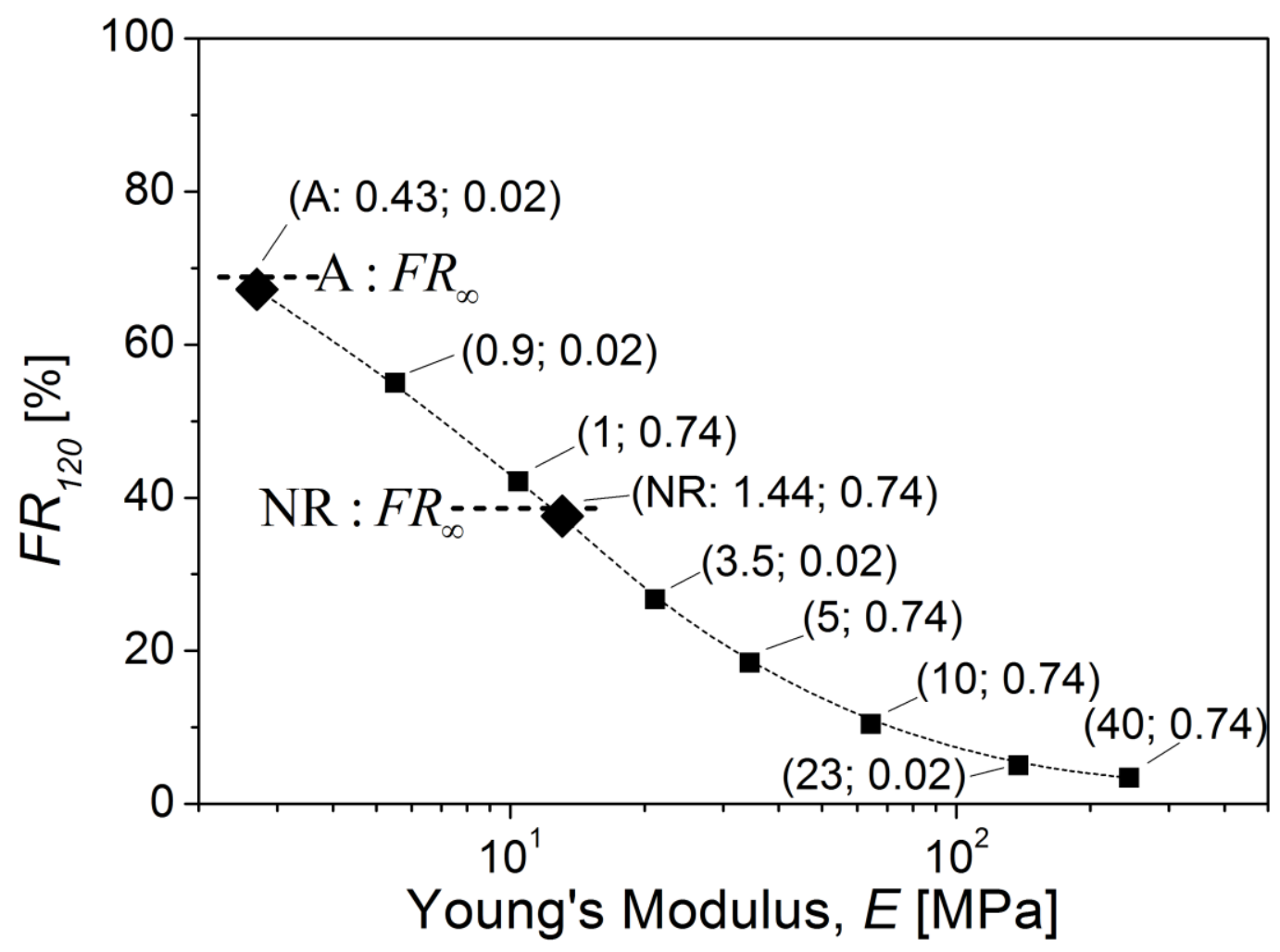

Fig. 13 
Table 1. Mooney-Rivlin's coefficients at stretch rate $60 \mathrm{~s}^{-1}$ (extrapolated values)

\begin{tabular}{lcccccc}
\hline Material & \multicolumn{3}{c}{$C_{10}[\mathrm{MPa}]$} & & \multicolumn{3}{c}{$C_{01}[\mathrm{MPa}]$} \\
& \cline { 2 - 7 } & & & & & \\
& $95 \%$ LPL & Mean & $95 \%$ UPL & $95 \%$ LPL & Mean & $95 \%$ UPL \\
\hline A & 0.28 & 0.43 & 0.59 & 0.00 & 0.02 & 0.06 \\
NR & --- & 1.44 & --- & 0.45 & 0.74 & 1.01 \\
\hline
\end{tabular}

LPL, UPL: lower and upper prediction limit, respectively 